\title{
Deviating to the right: Using eyetracking to study the role of attention in navigation asymmetries
}

\author{
Joanne S. Robertson • Jason D. Forte • Michael E. R. Nicholls
}

Published online: 17 December 2014

(C) The Psychonomic Society, Inc. 2014

\begin{abstract}
The ability to navigate accurately through the environment and avoid obstacles is essential for effective interactions with the environment. It is therefore surprising that systematic rightward errors are observed when neurologically intact participants navigate through doorways - most likely due to the operation of biases in spatial attention. These rightward errors may arise due to the operation of an extinction-like process, whereby participants overattend to the left doorpost and collide with the right one. Alternatively, rightward biases might reflect a bisection bias, such that the extrapersonal nature of the aperture causes participants to misbisect the aperture slightly to the right of true center. Because eye movements and spatial attention are closely related, in this study we used eyetracking to test the extinction and bisection models in a remote wheelchair navigation task. University students ( $n=16)$ made rightward errors when navigating the wheelchair through a doorway, and fixated more frequently toward the right side of the aperture throughout the trial. These results are inconsistent with an extinction-based theory of navigation asymmetry, which predicts a leftward bias in eye position due to participants overattending to the left side of the doorway. Instead, the observed rightward bias in eye movements strongly supports a bisection-based theory of navigation asymmetry, whereby participants mentally "mark" the midpoint of a doorway toward the right and then head toward that point, resulting in rightward deviations. The rightward nature of participants'
\end{abstract}

\section{J. S. Robertson · J. D. Forte}

University of Melbourne, Parkville, Victoria, Australia

J. S. Robertson $(\square)$

Florey Institute of Neuroscience and Mental Health, Parkville,

Victoria, Australia

e-mail: joanner@unimelb.edu.au

M. E. R. Nicholls

Flinders University, Adelaide, South Australia, Australia navigation errors and eye positions is consistent with the existence of a rightward attentional bias for extrapersonal stimuli.

Keywords Attention - Spatial cognition · Neglect . Pseudoneglect $\cdot$ Extrapersonal $\cdot$ Peripersonal $\cdot$ Eye movements $\cdot$ Navigation $\cdot$ Perceptual asymmetry

Unilateral spatial neglect provides a particularly salient example of the lateralized nature of spatial attention. Occurring most often as a consequence of parietal lesions in the right hemisphere (Bisiach \& Vallar, 1988; Molenberghs, Sale, \& Mattingley, 2012), neglect incorporates a number of dissociable clinical syndromes characterized by a failure to spontaneously orient, attend, and respond to leftward features of objects or space (Bartolomeo \& Chokron, 1999; Heilman, Watson, \& Valenstein, 2003; Robertson \& Halligan, 1999). Neglect can occur in a variety of sensory modalities (Brozzoli, Dematte, Pavani, Frassinetti, \& Farnè, 2006) and is modified as the stimulus moves from peri- to extrapersonal space (Aimola, Schindler, Simone, \& Venneri, 2012; Brain, 1941).

Symptoms of neglect are evident on a wide variety of clinical tasks, including line bisection (Bisiach \& Vallar, 1988; Heilman, Jeong, \& Finney, 2004; Heilman \& Valenstein, 1979; Toth \& Kirk, 2002), target cancelation (Weintraub \& Mesulam, 1987, 1988), and judgments of relative luminosity (Mattingley et al., 2004; Nicholls \& Roberts, 2002), numerosity (Luh, 1995), and size (Mayo et al., 1999; Nicholls, Bradshaw, \& Mattingley, 1999). Neglect can also significantly interfere with the capacity to perform routine activities of daily living (Azouvi et al., 1996), such as navigating through the environment. Because many neglect patients are unable to ambulate independently (Jackson, Thornton, \& TurnerStokes, 2000), most studies of navigation in neglect have examined the navigation proficiency of patients in wheelchairs. Studies of unilateral collisions in neglect have shown that 
patients disproportionately collide with objects on their unattended left side (Punt, Kitadono, Hulleman, Humphreys, \& Riddoch, 2008, 2011; Qiang, Sonoda, Suzuki, Okamoto, \& Saitoh, 2005; Turton et al., 2009; Webster et al., 1989; Webster, Rapport, Godlewski, \& Abadee, 1994; Webster et al., 1995). Studies examining veering trajectories, however, have shown mixed results. Wheelchair-bound neglect patients have been variously reported to veer leftward (Turton et al., 2009) and rightward (Punt et al., 2008; Robertson, Tegnér, Goodrich, \& Wilson, 1994), whilst ambulatory neglect patients have been observed to veer toward the right (Berti et al., 2002; Turton et al., 2009).

Although neglect patients fail to attend to stimuli on the left, neurologically healthy participants overattend the left side of space and show a subtle "pseudoneglect" to the right (Bowers \& Heilman, 1980). Pseudoneglect is influenced by many factors also known to moderate neglect, and like neglect it affects multiple sensory modalities (Baek et al., 2002; Vallar, Guariglia, Nico, \& Bisiach, 1995) and regions of space (Drain \& Reuter-Lorenz, 1996; Longo \& Lourenco, 2006). For these reasons, it is assumed that neglect and pseudoneglect are closely related in terms of their underlying cognitive and neurological mechanisms (McCourt \& Jewell, 1999; Nicholls, Mattingley, \& Bradshaw, 2005).

Pseudoneglect can be quantified with the same clinical assessment tasks used to measure neglect, such as the line bisection (Jewell \& McCourt, 2000) and grayscale (Nicholls et al., 1999; Nicholls, Smith, Mattingley, \& Bradshaw, 2006) tasks, on which subtle leftward biases are evident. Interestingly, when tasks are presented in participants' extrapersonal space, these leftward biases reverse to a rightward bias (Gamberini, Seraglia, \& Priftis, 2008; Heber, Siebertz, Wolter, Kuhlen, \& Fimm, 2010; Longo \& Lourenco, 2006).

Like neglect, pseudoneglect affects navigation asymmetries, albeit in a more subtle fashion. Using a self-report design, Turnbull and McGeorge (1998) asked students to recall whether they had collided with anything recently, and on which side. The researchers found a trend for more reports of collisions to the right, which was related to line bisection asymmetries. Subsequent laboratory-based experiments have largely confirmed the predominance of right-sided collisions or veering when participants walk (Nicholls, Loftus, Mayer, \& Mattingley, 2007; Nicholls, Loftus, Orr, \& Barre, 2008) or drive a vehicle (Jang, Ku, Na, \& Lee, 2009; Nicholls, Hadgraft, et al., 2010) through doorways (but cf. Fujikake, Higuchi, Imanaka, \& Maloney, 2011; Hatin, Tottenham, \& Oriet, 2012).

A number of mechanisms have been proposed to account for rightward navigation biases. One possibility is that the rightward bias may reflect underlying motor asymmetries (Boyadjian, Main, \& Danion, 1999; Fujikake et al., 2011) or turning biases mediated by a vestibular (Robertson et al., 1994) or dopaminergic (Mohr, Landis, Bracha, Fathi, \& Brugger, 2003; Mohr, Landis, Bracha, Fathi, \& Brugger, 2005) asymmetry. Although these motor/vestibular mechanisms could explain asymmetries for ambulatory tasks, they have difficulty explaining asymmetries when navigating a motorized wheelchair (Nicholls, Hadgraft, et al., 2010) or in virtual-reality paradigms (Jang et al., 2009; Kim et al., 2010; Thomas, Stuckel, Gutwin, \& Elias, 2009)-because these tasks operate independently of the motor effectors. In addition, vestibular effects on veering and turning behavior are typically observed only when participants are blindfolded (Cohen, 2000; Roberts, Cohen, \& Sangi-Haghpeykar, 2011).

A better explanation of navigation asymmetries is provided by the lateralized mechanisms that control spatial attention (see Hatin et al., 2012; Nicholls, Hadgraft, et al., 2010). One possibility is that navigation asymmetries are related to an extinction-like process. In clinical neglect, extinction is characterized by impaired detection (i.e., "extinction") of stimuli in the contralesional hemispace under conditions of bilateral stimulation (de Haan, Karnath, \& Driver, 2012; Verfaellie \& Heilman, 2005). A similar process may operate during aperture navigation. If the two doorposts compete for attention, the overattended doorpost should be more effectively avoided than the underattended doorpost, resulting in more frequent collisions with the latter. In the case of neglect patients, who overattend to the right (Heilman et al., 2003; Robertson \& Halligan, 1999), this model would predict more collisions with the left doorpost. Neurologically healthy participants, in contrast, would overattend to the left doorpost as they approach the aperture (due to the operation of pseudoneglect in peripersonal space), resulting in collisions with the right doorpost.

An extinction-based theory of navigation asymmetries is supported by findings that navigation errors are modulated by unilateral cues (Fujikake et al., 2011) and motor activity (Nicholls et al., 2007), both of which cause an imbalance in the distribution of attentional resources (Kinsbourne, 1970, 1977, 1987). An extinction-like process cannot easily account, however, for research showing that navigation errors persist when participants navigate through an implicit "aperture" denoted by markers on the floor rather than through explicit doorposts (Nicholls, Hadgraft, et al., 2010), or the observation that increasing the width of apertures increases the magnitude of rightward navigation errors (Berti et al., 2002; Nicholls, Hadgraft, et al., 2010). Both of these studies suggest that the space separating the doorposts is what is important, rather than the doorposts themselves.

Spatial attention could also affect navigation asymmetries through a bisection bias (Nicholls, Hadgraft, et al., 2010). Line bisection errors occur toward the end of a stimulus that is overattended (Bradshaw, Bradshaw, Nathan, Nettleton, \& 
Wilson, 1986; Bultitude \& Aimola Davies, 2006; McCourt \& Jewell, 1999). In the case of a door, which is located in extrapersonal space, the perceived midpoint would be located to the right of the true center (Longo \& Lourenco, 2006). Research by Berti at al. (2002) has demonstrated that participants mentally "mark" the center of an aperture in extrapersonal space and then head for that mark without updating their estimate. It is therefore possible that participants mentally mark the center of the doorway slightly to the right of true center, and then head toward that point — resulting in rightward deviations and bumping.

A bisection-based theory of navigation asymmetry is supported by findings that both navigation and bisection errors are affected in similar ways by manipulations of factors such as cueing (Hatin et al., 2012; Robertson et al., 1994) and aperture width (Berti et al., 2002; Nicholls, Hadgraft, et al., 2010). The link between navigation and bisection asymmetries can also be tested through correlational analyses. Although some studies have shown an association between biases in line bisection and subsequent navigation/bumping asymmetries (Kim et al., 2010; Nicholls et al., 2008; Turnbull $\&$ McGeorge, 1998) others have shown no relationship between the tasks (Nicholls, Hadgraft, et al., 2010; Nicholls, Loetscher, \& Rademacher, 2010; Nicholls et al., 2007).

A final possibility that needs to be considered relates to cultural practices associated with asymmetries in driving habits. It is known that cultural practices, such as reading direction, can affect perceptual asymmetries (Chokron, Bartolomeo, Perenin, Helft, \& Imbert, 1998; Chokron \& Imbert, 1993; but cf. McCourt, 2001; Nicholls \& Roberts, 2002). It is therefore possible that the side of the road on which people drive affects navigation asymmetries. How road rules affect navigation asymmetries is a matter of debate. Nicholls, Hadgraft, et al. (2010) suggested that, if road rules were important, participants should deviate toward the side on which they drive. In the case of Australian drivers, who drive on the left side of the road, this is the opposite of the rightward deviation that has been observed (Nicholls, Hadgraft, et al., 2010). The potential impact of driving rules will be elaborated further in the Discussion section of this article.

Rightward deviations when navigating an aperture are a surprising, but poorly explained, behavioral phenomenon. Given the current uncertainty in relation to the cause of this phenomenon, in this study we tested the predictions of the extinction and bisection models in a group of neurologically intact individuals using a wheelchair navigation task combined with eyetracking. Participants were asked to navigate a wheelchair through a doorway using a remote control. Remote control was used because it kept the doorway at a constant distance, and therefore any changes in eye position throughout the trial could not be due to changes in peripersonal/extrapersonal space or visual angle. In addition, remote control suited the available eyetracking technology, which needed to be fixed in relation to the doorway. As Nicholls, Hadgraft, et al. (2010) observed, it was expected that participants would deviate to the right as the wheelchair passed through the door.

Participants' eye movements were monitored as they performed the navigation task. A strong link is known to exist between attention and eye movements (Beauchamp, Petit, Ellmore, Ingeholm, \& Haxby, 2001; Deubel \& Schneider, 1996), and this is clearly evident in neglect patients, whose eye movements are confined almost exclusively to the right when searching for a target in darkness (Hornak, 1992; Karnath \& Fetter, 1995).

The extinction and bisection models of navigation asymmetry make quite different predictions for the pattern of eye movements. The extinction model suggests that too much attention is paid to avoiding the left doorpost and, as a consequence, participants deviate or collide to the right. If this model is correct, there should be more fixations on the left side of the doorway. Some evidence for this proposal has come from a study by Cinelli, Patla, and Allard (2009). They asked participants to walk down a pathway and pass through a doorway, which opened and closed at a number of different frequencies. Although Cinelli et al. reported accuracy relative to the center of the doorway, they unfortunately did not measure deviations to the left or right. Cinelli et al., however, did report asymmetries in eye position, and found that participants directed more fixations toward the left than toward the right door. Interestingly, Cinelli et al. related this fixation asymmetry to driving habits rather than to lateralization related to brain function and attention. They suggested that their North American population are accustomed to passing cars and people to their left, and therefore fixate and pay more attention on this side. It can be seen, nevertheless, that the leftward bias in eye position also supports the extinction model.

The bisection model makes a different set of predictions. In this case, participants misbisect the door slightly to the right of true center and then head toward this point. The bisection model would therefore predict more fixations to the right. To analyze asymmetries in the pattern of eye movements, the mean horizontal eye position throughout the trial was calculated. To assess whether asymmetries in eye movements changed as the wheelchair approached the door, a time analysis was performed. Although Berti et al. (2002) proposed that walking trajectories are not updated as the participant moves toward the target, it is also possible that changes would be observed as the wheelchair moved from extra- to peripersonal space. A correlational analysis was conducted to determine whether an association existed between where participants were looking and where they bisected the aperture. 
Although a wheelchair operated via remote control provides an excellent means of investigating navigation asymmetries in near and far space, it does contain asymmetries in its own right. For example, it is possible that a mechanical asymmetry in the wheelchair could cause it to "pull" slightly to the left or right. It is also possible that participants might imagine themselves sitting in the wheelchair as they controlled it and follow road rule conventions. In the case of Australia, this would mean driving on the left side. To control for both of these factors, the wheelchair was turned around for half of the trials, so that the wheelchair was driven either forward or backward through the aperture. If mechanical and/or driving factors were important, we should observe a difference between the forward and backward conditions.

\section{Method}

Participants

Sixteen undergraduate students (seven male, nine female) participated in this experiment as part of their course requirements. They were strongly right-handed, as measured by Oldfield's (1971) Edinburgh Inventory (handedness quotient: $M=86.88, S D=16.62)$, and they ranged in age from 18 to 42 years $(M=20.50, S D=6.01)$.

\section{Apparatus and stimuli}

The aperture was composed of eight stackable plastic crates (the $x, y$, and $z$ dimensions were all $400 \mathrm{~mm}$ ). Four crates were stacked on top of one another to create two towers that were each 1,600 mm high (see Fig. 1). The towers were positioned such that they were separated by a distance of $1,100 \mathrm{~mm}$, through which participants could navigate the wheelchair. A spirit level was used to ensure that both towers were exactly perpendicular to the floor. The motorized wheelchair navigated by the participants (model: Glide Rehabilitation Products, Power-Glide Series 4, 17 in.) was similar to those used by people with mobility impairments. The wheelchair was controlled using a joystick. For the purposes of the present study, the joystick was removed from the wheelchair and connected to a long cable, such that participants were able to navigate the vehicle remotely. A thin white line down the front and rear of the chair's back rest marked the center for scoring purposes. An Apple iMac computer interfaced with MATLAB software (MathWorks) and the OpenGL-based Psychophysics Toolbox extension (version 3; Brainard, 1997) was used to administer the experiment. Eye position was recorded using a headmounted infrared eyetracking system (EyeLink II, SR Research) with 500-Hz binocular eye monitoring and a 0.5 deg average accuracy. This eyetracking equipment is designed for use in experiments that present stimuli on a standard computer monitor, meaning that its calibration paradigm involves directing participants to fixate on particular points on a computer monitor with a screen resolution of $1,024 \times$ 768 pixels. To accommodate the fact that participants were attending to a doorway and not a computer screen, the calibration process was modified by constructing an enlarged calibration "screen," upon which nine (three rows by three columns) calibration targets were printed inside a rectangular array $1,024 \mathrm{~mm}$ wide and $768 \mathrm{~mm}$ high. The screen was placed across the front of the aperture, precisely centered, for the calibration process at the outset of the experiment. The position of the screen was kept constant for all participants, regardless of their height, because up/down eye movements were not of interest in this experiment.

\section{Procedure}

The experiment was conducted in a small classroom, which had been cleared of any stimuli that could cue participants' attention toward either side of the aperture. Participants were seated in a chair immediately behind the starting position of the vehicle, aligned with the center of the aperture. A tripod supporting a digital video camera (model: JVC Everio GZ-MG330) was situated directly behind the participant's chair, also aligned with the center of the aperture. Video recording was captured at $4.2 \mathrm{MBps}$, at a resolution of $720 \times 480$ pixels. The experiment began with the fitting of the eyetracking equipment and the calibration screen. During the calibration process, participants were instructed by the experimenter to look at each target in accordance with the order stipulated by an automated calibration schedule. The accuracy of calibration across the $x$ (horizontal) axis was emphasized over the $y$ (vertical) axis, since the primary interest of this study was left-right asymmetries in eye position. The calibration screen was removed from the aperture once adequate calibration was achieved.

To control for any effects related to steering hand, half of the participants steered the vehicle with their left hand and half with their right. Although bimanual control may have been preferable, pretests showed that participants tended to favor one hand over the other, despite the instructions. Each trial began with the front wheels of the vehicle positioned in a marked "starting position," which ensured that the midline of the vehicle was aligned with the center of the aperture. Video recording of the trial commenced, and participants were asked to maneuver the vehicle all the way through the aperture. Participants were asked to attempt to line up the vehicle such that it passed through the aperture "exactly in the middle" of the two posts. Following each trial, the vehicle was returned to 

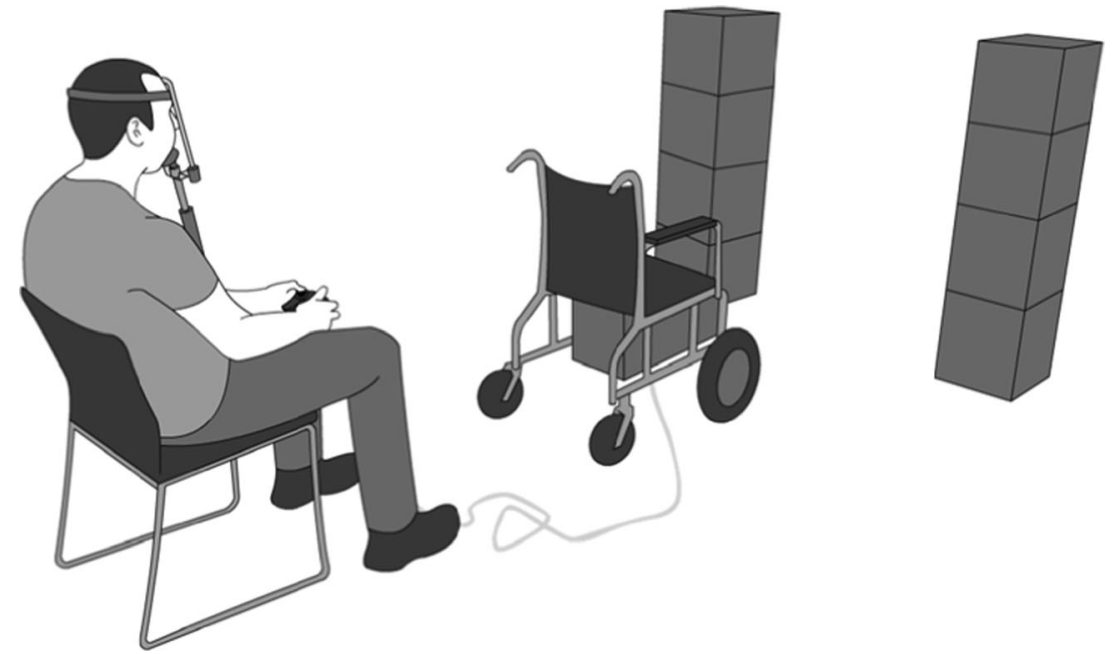

Fig. 1 Diagram of the experimental setup, showing the participant guiding a wheelchair through an aperture using a remote control. The camera behind the participant is not shown

its starting position in preparation for the next trial. The experimenter pressed a key at the beginning and end of each navigation trial to indicate the period of time over which the eye movement data were relevant. Participants always completed at least five practice trials to ensure familiarity with the procedure prior to commencement of the experimental trials. In the event that participants collided with any part of the aperture, the trial was discarded and repeated. This was done because any collision with the aperture was likely to move the sides of the doorway - meaning that they had to be reset. Participants completed 40 trials in total: 20 with the wheelchair facing backward and 20 with it facing forward. The order of blocks was counterbalanced across participants. The side on which the control cable lay relative to the wheelchair was also balanced between participants. Following the completion of the navigation trials, the calibration screen was returned to the aperture and the accuracy of calibration of the eyetracker was confirmed to ensure that no significant degradation had occurred throughout the experiment.

\section{Data analysis}

Video clips were first processed to determine participants' deviations from the objective center of the aperture in each navigation trial. VLC Media Player 1.0 software was used to take a static snapshot of the position of the wheelchair as it passed through the aperture. The distance of the midline of the vehicle from the midline of the aperture was then measured (in pixels) using GIMP 2.6.7 software, before being converted to millimeters via a conversion ratio computed using the width of the aperture in pixels relative to the width of the aperture in millimeters. Values to the left of center were scored as negative, and values to the right were scored as positive. This preliminary processing was primarily completed by the first author, but to ensure objectivity in measurement, approximately $20 \%$ was completed by research assistants who were naïve to the specific aims of the experiment. Consistency between the measurements of different raters was good: Interrater reliability was .88 for the deviation scores of all trials for a randomly selected participant.

With regard to the eyetracking data, calibration at the end of the experiment indicated no major departures from the initial calibration for any participant. Due to technical difficulties during the transfer of eyetracking information, the data for two participants were incomplete. One participant was missing all trials in the backward condition, and another was missing the final ten trials in the backward condition. Given the nonrandom nature of the missing data, it was not feasible to estimate the missing values, so the analysis was performed on the available data for these participants.

Because of the large volume of data collected by the eyetracker, it was necessary to summarize it prior to analysis. The length of trials varied between approximately 5 and $20 \mathrm{~s}$, which resulted in over 5,000 eye position samples for some trials. To condense the data into a more manageable form, the mean gaze position along the $x$ (horizontal) axis was computed for each trial. The data were scaled in millimeters to allow for direct comparison with the navigation data. It is important to note, however, that the possible magnitude of gaze deviations from center was much larger than the possible magnitude of navigation deviations, since the extent of the latter was limited by the physical presence of the "doorposts" between which participants were obliged to navigate the wheelchair. No constraints were put on the possible magnitude of eye 


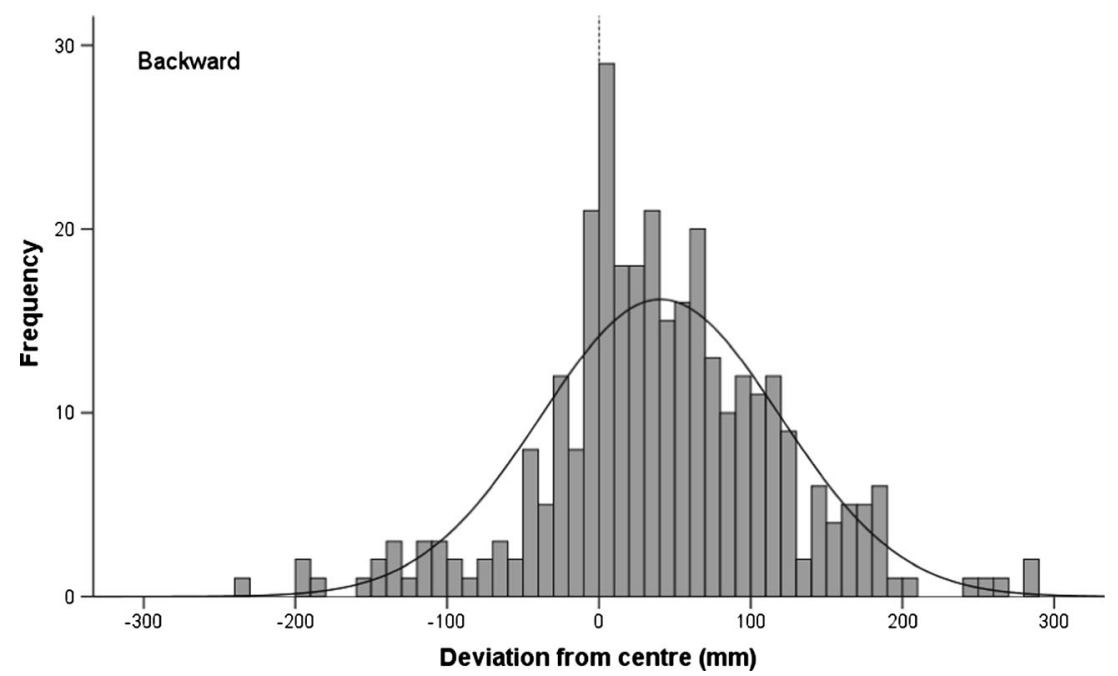

Fig. 2 Navigation deviations from center for the 320 (16 participants $\times 20$ trials) data points in the backward condition. Bars reflect 10-mm response categories. Negative and positive values denote leftward and rightward deviations, respectively

movements, however, meaning that they could equate to deviations from the center of the aperture well in excess of its physical boundaries.

To examine the distribution of participants' attention throughout the navigation task, the eyetracking data were also examined in terms of their pattern across time. Time series data for each trial were constructed by scaling the length of the trial from 0 to 1 to indicate the proportion of the trial that had elapsed when each recording of eye position occurred. In cases in which multiple eye positions were recorded with the same time stamp, the eye position values were averaged to provide a single eye position at each time. A spline function was fitted to these data points, and then eye position values from 100 equally spaced time intervals were interpolated from the spline function. This method ensured that each trial was equally weighted, instead of overweighting longer trials by including more data points from them. The data for each time interval (1 to 100) were then averaged across all trials in each condition, to give an indication of the participants' general patterns of eye movements across time. The mean eye positions of individual participants at each of the 100 time intervals across the 30 trials were also computed, to investigate whether different individuals displayed different patterns of eye movements throughout the task.

\section{Results}

\section{Navigation data}

The distributions of navigation errors across all trials and participants in the backward and forward conditions are shown in Figs. 2 and 3, respectively. Participants deviated to the right in both the backward $(M=40.85 \mathrm{~mm}, S D=27.25)$

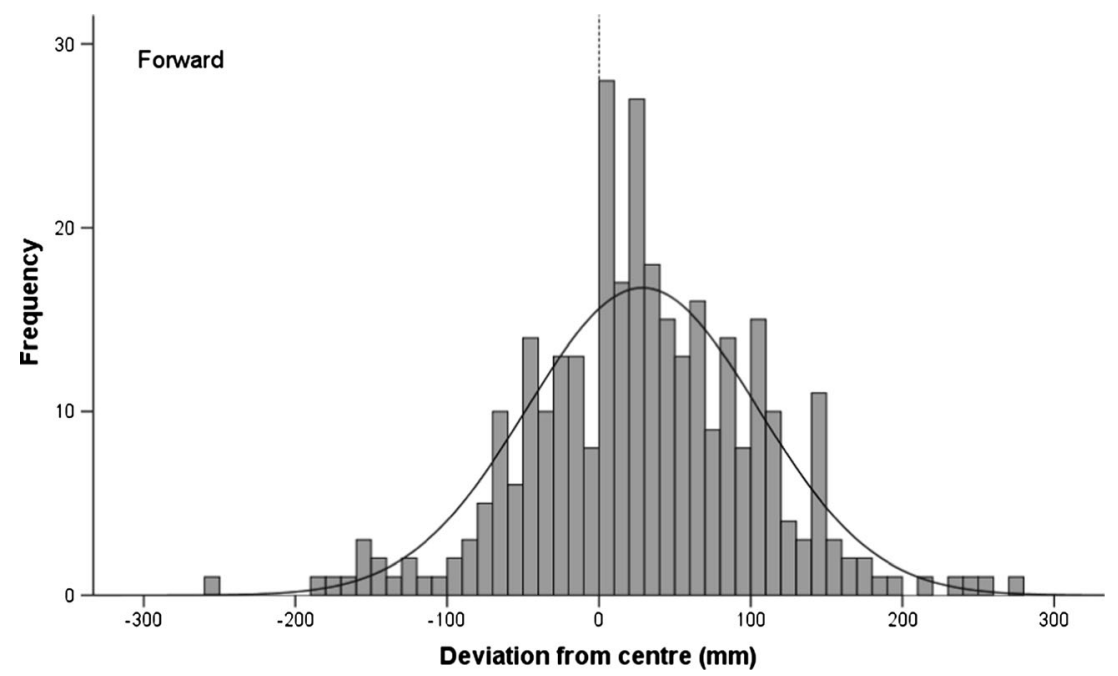

Fig. 3 Navigation deviations from center for the 320 (16 participants $\times 20$ trials) data points in the forward condition. Bars reflect 10 -mm response categories. Negative and positive values denote leftward and rightward deviations, respectively 


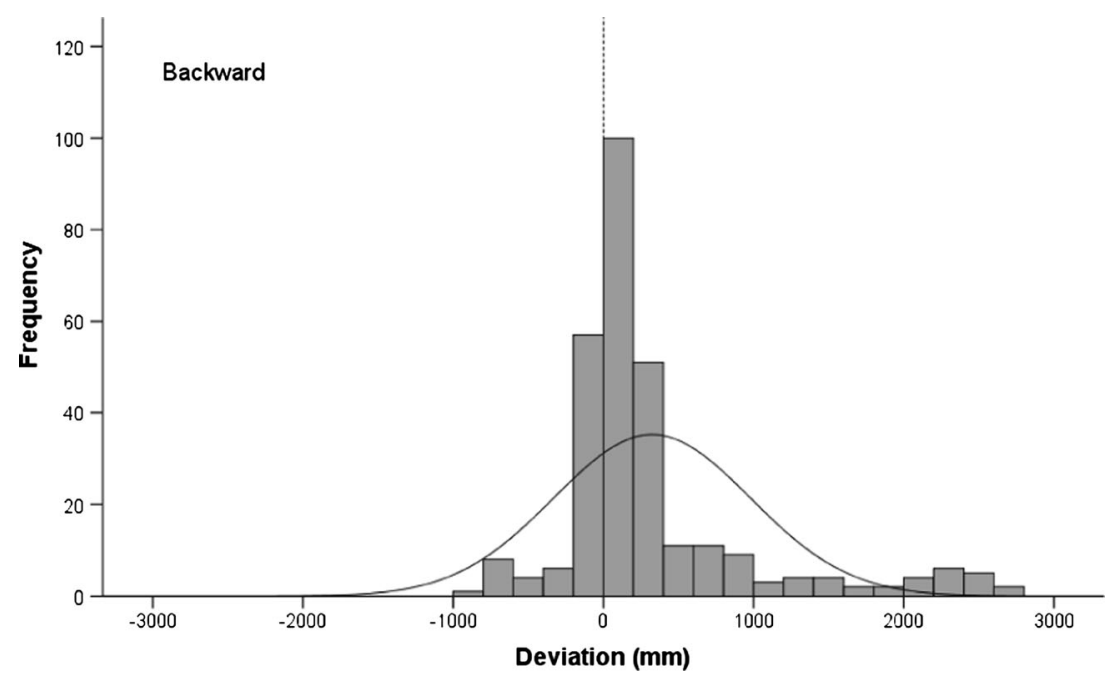

Fig. 4 Mean eye positions in each of 290 trials in the backward condition. Bars reflect 200-mm response categories. Negative and positive values denote leftward and rightward gaze positions, respectively

and forward $(M=26.17 \mathrm{~mm}, S D=26.97)$ conditions. Singlesample $t$ tests confirmed that the rightward deviations differed significantly from zero for both conditions [backward, $t(15)=$ $6.00, p<.001$, Cohen's $d=1.50$; forward, $t(15)=3.88, p=$ .001 , Cohen's $d=0.97]$. A paired-samples $t$ test showed no effect of the wheelchair's orientation on navigation asymmetry $[t(15)=1.58, p=.13$, Cohen's $d=0.54]$.

\section{Eyetracking data}

Histograms showing participants' mean eye positions for all trials in the backward and forward conditions are shown in Figs. 4 and 5.

Participants' mean eye position throughout the trials was to the right in both the backward $(M=318.98 \mathrm{~mm}, S D=622.69)$ and forward $(M=305.05 \mathrm{~mm}, S D=547.83)$ conditions. Single-sample $t$ tests against $0 \mathrm{~mm}$ (no bias) showed that this deviation approached significance in the backward condition $[t(14)=1.98, p=.07]$ and was statistically significant in the forward condition $[t(15)=2.23, p=.04$, Cohen's $d=0.56]$. When the data were averaged across both the backward and forward conditions, the bias was also significantly to the right $(M=306.62 \mathrm{~mm}, S D=569.37)[t(15)=2.15, p=.05$, Cohen's $d=0.50]$. A paired-samples $t$ test showed no difference in the magnitudes of the biases between the backward and forward conditions $[t(15)=0.07, p=.94]$.

Figure 6 displays the mean gaze positions of the individual participants in the two conditions. Most participants showed a slight bias toward the right of the aperture, whereas a small number (DL, EC, and perhaps YC) showed much larger rightward biases.

Figure 7 displays all participants' patterns of eye movements over time across all trials. The mean eye positions of individual participants over time across all trials are displayed

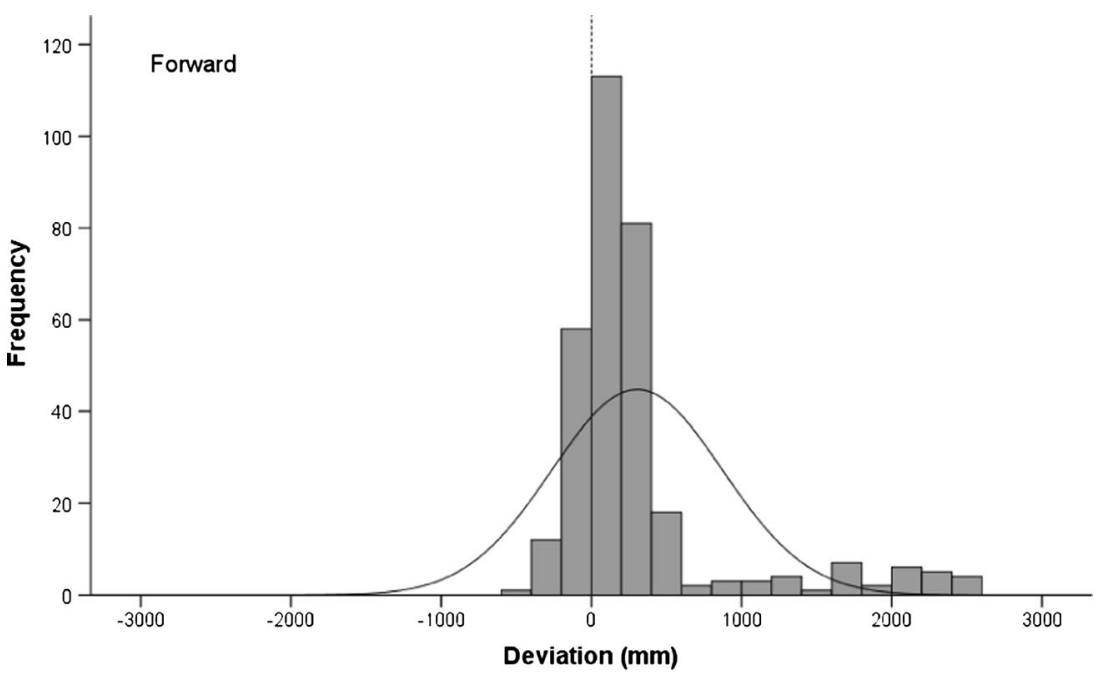

Fig. 5 Mean eye positions in each of 320 trials in the forward condition. Bars reflect 200-mm response categories. Negative and positive values denote leftward and rightward gaze positions, respectively 


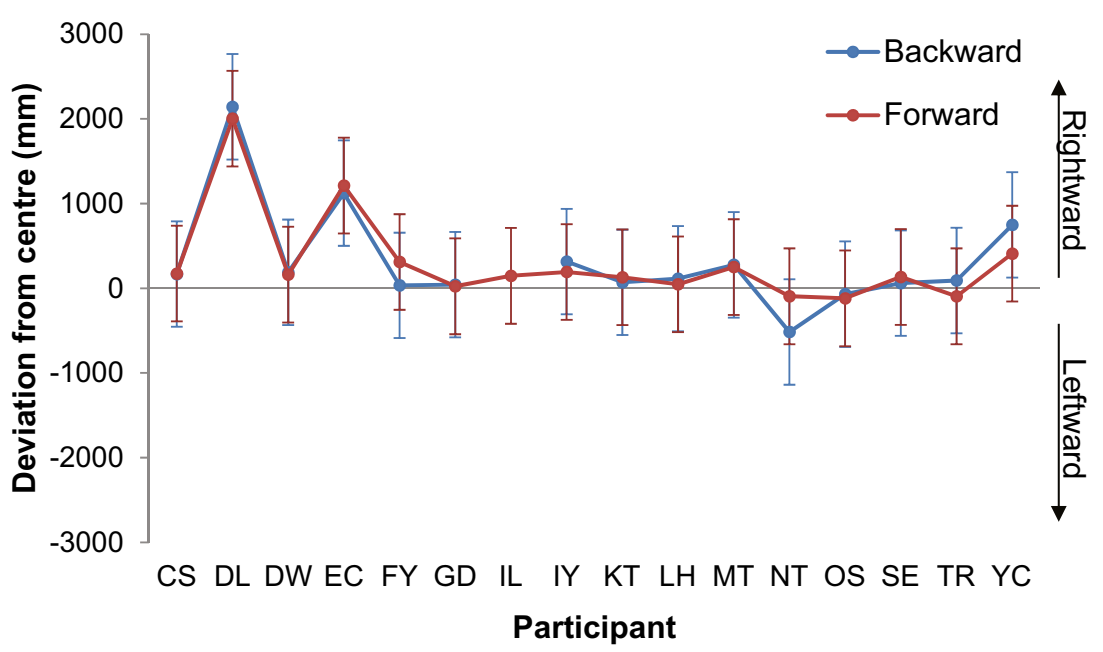

Fig. 6 Mean eye positions $( \pm 1 S D)$ of individual participants as a function of wheelchair orientation. Negative and positive scores indicate leftward and rightward gaze directions, respectively

for the backward and forward conditions in Figs. 8 and 9, respectively.

It is clear from Fig. 7 that, on average, participants looked toward the right side of the aperture for the entire duration of the navigation trials. There is no indication that participants' patterns of eye movements differed in later parts of the trials, when the wheelchair approached the aperture, relative to earlier parts. That said, Figs. 8 and 9 show considerable differences between individuals in terms of the directions in which they tended to look. Most participants displayed a rightward eye position bias, characterized by them typically looking somewhere between the center of the aperture and the right doorpost, though a smaller number of participants showed similar biases toward the left side. A small minority of participants (DL, EC, and YC in the backward condition, and DL and EC in the forward condition) demonstrated extreme rightward biases, characterized by them looking beyond the right doorpost throughout the majority of the navigation task.

Although there were reasons to explain the large rightward biases in eye position (see the Discussion section), the data were reanalyzed after removing DL, EC, and $\mathrm{YC}$ in the backward condition and DL and EC in the forward condition. The results were basically the same. The rightward bias in the backward condition failed to reach statistical significance $(M=64.22 \mathrm{~mm}, S D=211.73)[t(11)=1.05, p=.31] . \mathrm{In}$ contrast, the rightward biases were significant for the forward $(M=118.98 \mathrm{~mm}, S D=154.84)[t(13)=2.87, p=.02$, Cohen's $d=0.60]$ and averaged $(M=106.85 \mathrm{~mm}, S D=$ 171.03) $[t(15)=2.33, p=.05$, Cohen's $d=0.57]$ conditions.

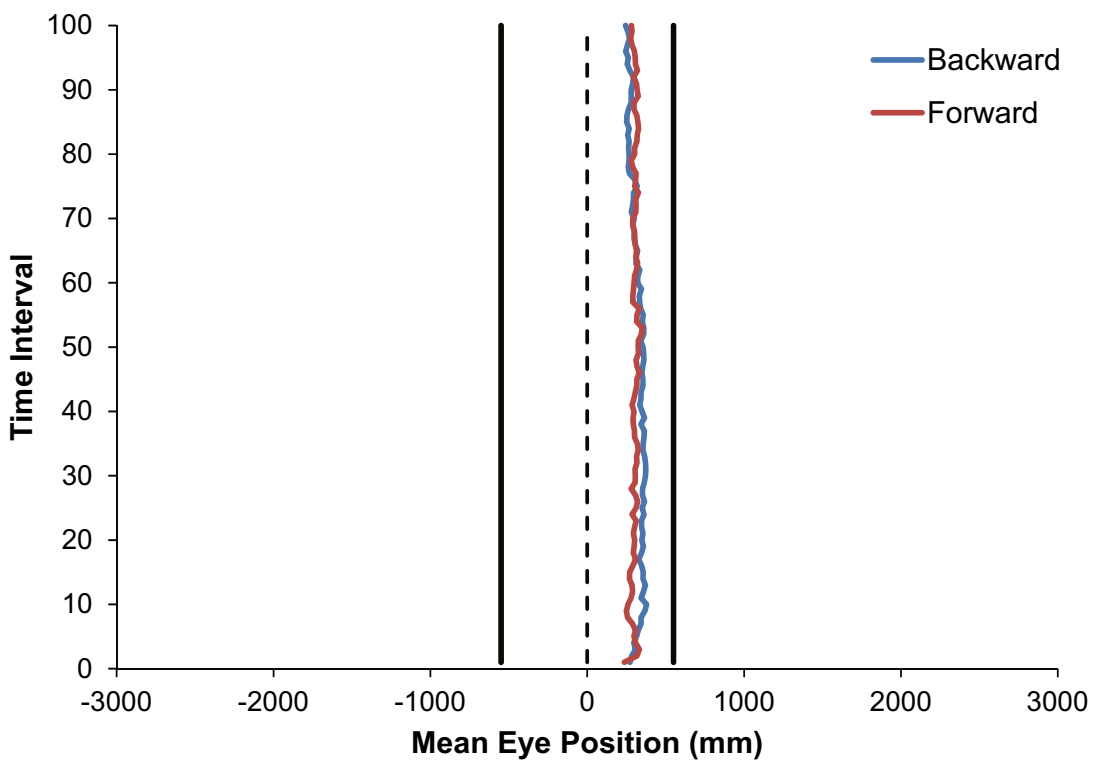

Fig. 7 Mean trajectories of participants' eye movements across time. Negative and positive scores denote leftward and rightward eye positions, respectively. The solid black lines represent the aperture doorposts 


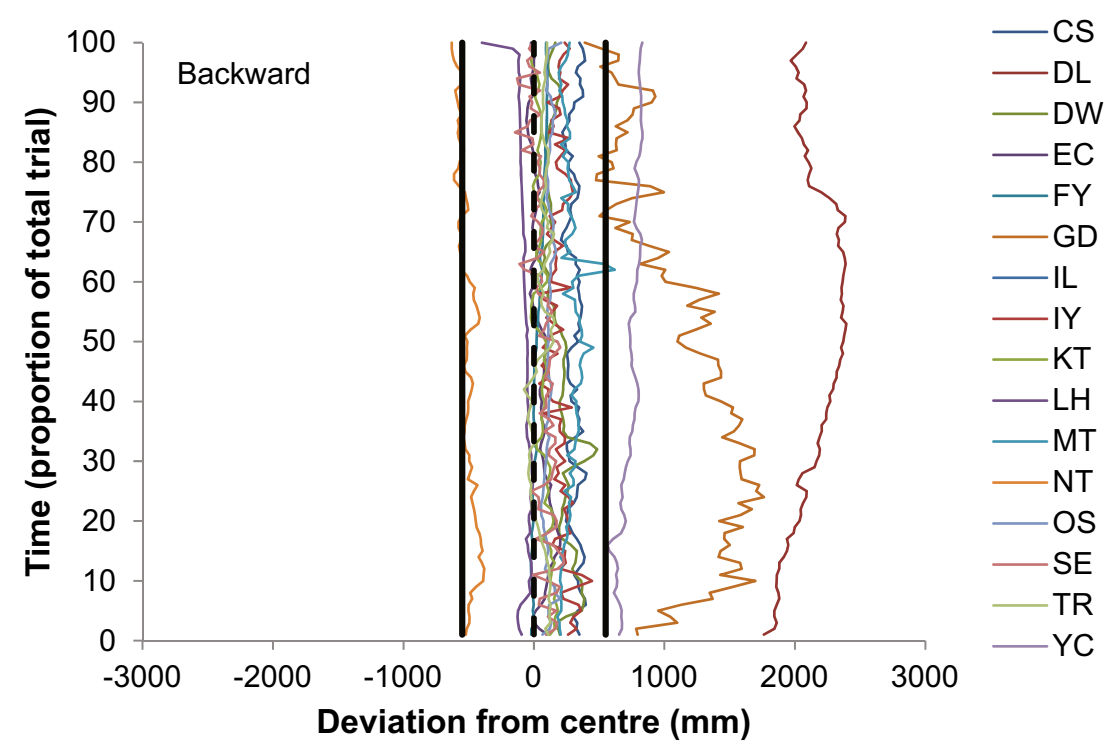

Fig. 8 Mean trajectories of individual participants' eye movements across time in the backward condition. Negative and positive values denote leftward and rightward eye positions, respectively. Each colored

We found no difference between the backward and forward conditions $[t(12)=0.56, p=.57]$.

\section{Relationship between navigation errors and eye position}

If biases in navigation and eye movements both reflect an underlying attentional asymmetry, it might be expected that the two measures would correlate. Pearson product-moment correlations were performed to investigate whether navigation errors were related to the mean eye position during each trial. The association approached significance in the backward condition, $r(290)=.116, p=.048$, but was not significant in line represents one participant; the solid black lines represent the aperture doorposts

the forward condition, $r(320)=-.063, p=.260$. The relationships between navigation error and eye position for the backward and forward conditions are shown in Figs. 10 and 11, respectively.

\section{Discussion}

In this experiment, we examined the mechanisms that give rise to asymmetries in navigation by examining the position of a motorized wheelchair as it passed through a doorway, combined with the position of eye fixations during the task.

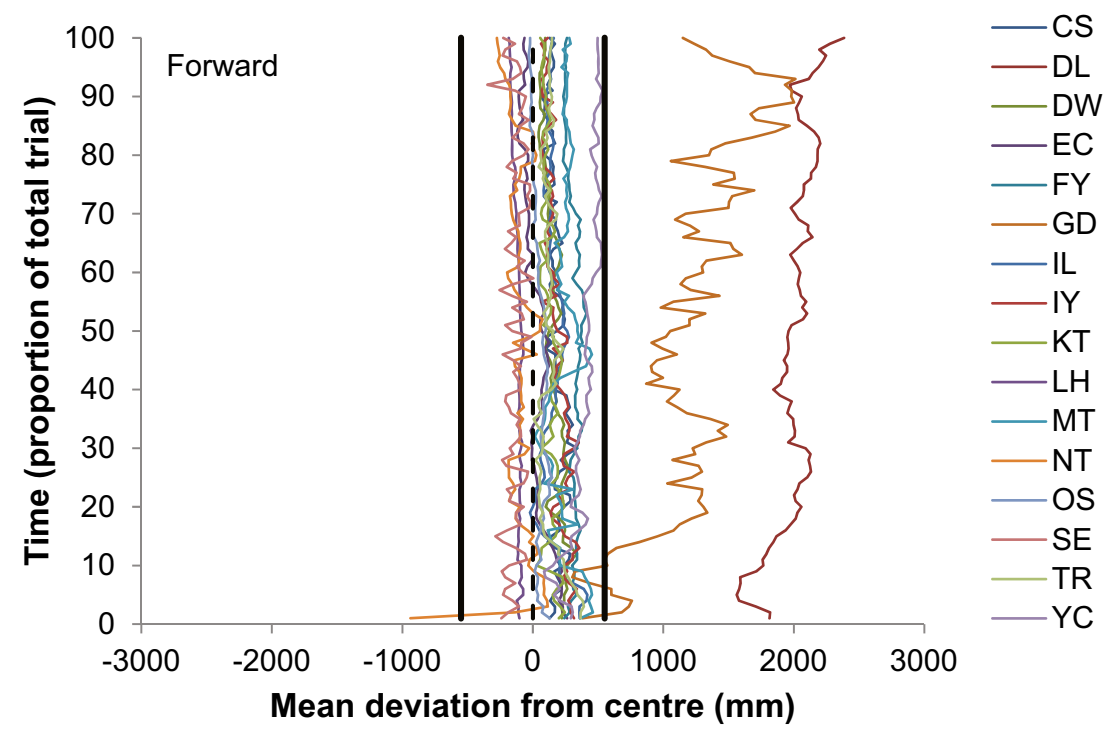

Fig. 9 Mean trajectories of individual participants' eye movements across time in the forward condition. Negative and positive values denote leftward and rightward eye positions, respectively. Each colored line represents one participant; the solid black lines represent the aperture doorposts 
Analysis of the position of the wheelchair revealed that participants navigated the wheelchair to the right of the true center as it passed through the doorway. This rightward deviation is consistent with a number of experiments that have shown rightward deviations for ambulatory tasks (Nicholls et al., 2007; Nicholls et al., 2008; but cf. Hatin et al., 2012), as well as for motorized wheelchairs (Nicholls, Hadgraft, et al., 2010) and simulated driving (Jang et al., 2009). Navigation errors were unaffected by the direction that the wheelchair was facing throughout the task, demonstrating that a mechanical asymmetry cannot explain the rightward deviation.

Our analysis of mean eye positions revealed that participants fixated toward the right side of the aperture. Although the paired-samples $t$ test revealed no significant difference between the forward and backward conditions, one-sample $t$ tests revealed that the rightward deviation was significant in the forward condition and only approached significance in the backward condition. The difference between the forward and backward conditions may therefore reflect a smaller sample size, which gave rise to increased variability in the backward condition $(S D=622.69 \mathrm{~mm})$ than in the forward condition $(S D=547.83)$. Throughout the time course of the trials, individual participants were very consistent in terms of the positions of their eyes (see Figs. 7-9). We found no indication that the side toward which participants attended changed as the wheelchair approached the doorway. This consistency is in accord with the model proposed by Berti et al. (2002) and suggests that participants select a point and then aim for that point throughout a trial.

The wheelchair and eye position data are inconsistent with an extinction-based theory of navigation asymmetry, which predicts a leftward bias in eye position due to participants overattending to the left side of the doorway. The results are also inconsistent with the data collected by Cinelli et al. (2009), who found that the majority of fixations were made to the left side of the aperture. There are a number of possible explanations for the discrepancy between the results of the present study and those reported by Cinelli et al. The most obvious difference between the studies relates to the dynamics of the aperture. Although the aperture was held constant in the present study, the width of the aperture changed constantly within each trial as the participant approached the doorway in Cinelli et al.'s study. In addition, the rate at which the aperture changed and the symmetry of the change was varied between trials. It is highly likely that the dynamics of the door affected the way that participants approached the tasks. In the present study, participants were able to identify a point within the aperture and then to head for that point without updating their trajectory - consistent with the bisection model. In contrast, the task used by Cinelli et al. required participants to constantly update their trajectory and speed. Evidence for this updating can be found in Cinelli et al.'s fixation data, which shows that

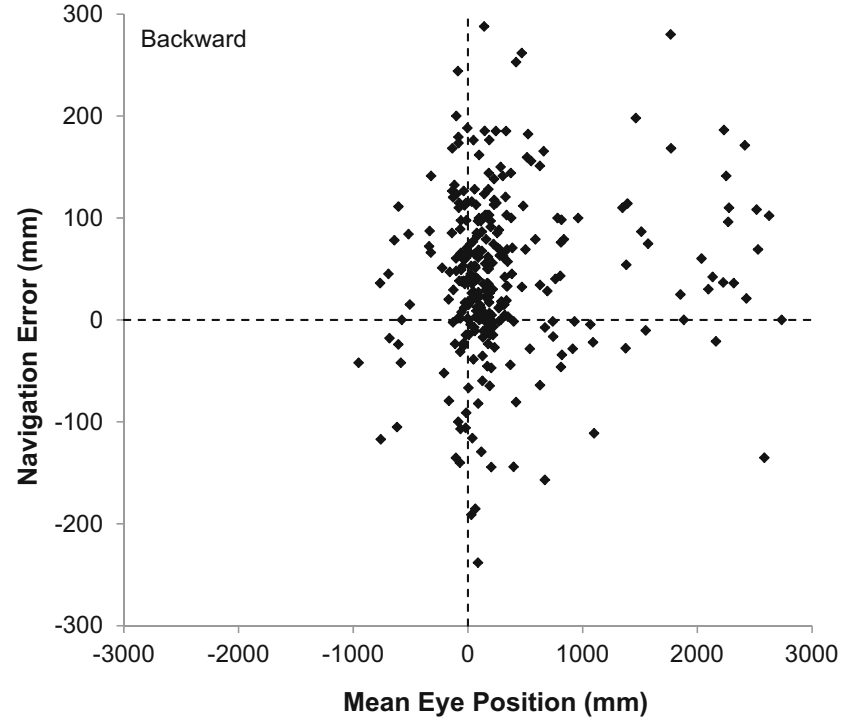

Fig. 10 Correlations between navigation errors and mean eye positions in the backward condition. Each data point represents one trial. Negative and positive values denote leftward and rightward deviations, respectively

the predominance of leftward fixations decreased as the participant approached the doorway. What caused the predominance of leftward fixations at the beginning of the trial is uncertain. It is interesting to note, however, that participants were required to walk along a relatively narrow walkway as they approached the door in the study by Cinelli et al. The study by Hatin et al. (2012), which found a leftward bias for bumping, also used a corridor on the approach to the aperture. It is therefore possible that the passageways used by Cinelli et al. and Hatin et al. changed the ways that participants coded space - perhaps bringing the aperture into near space.

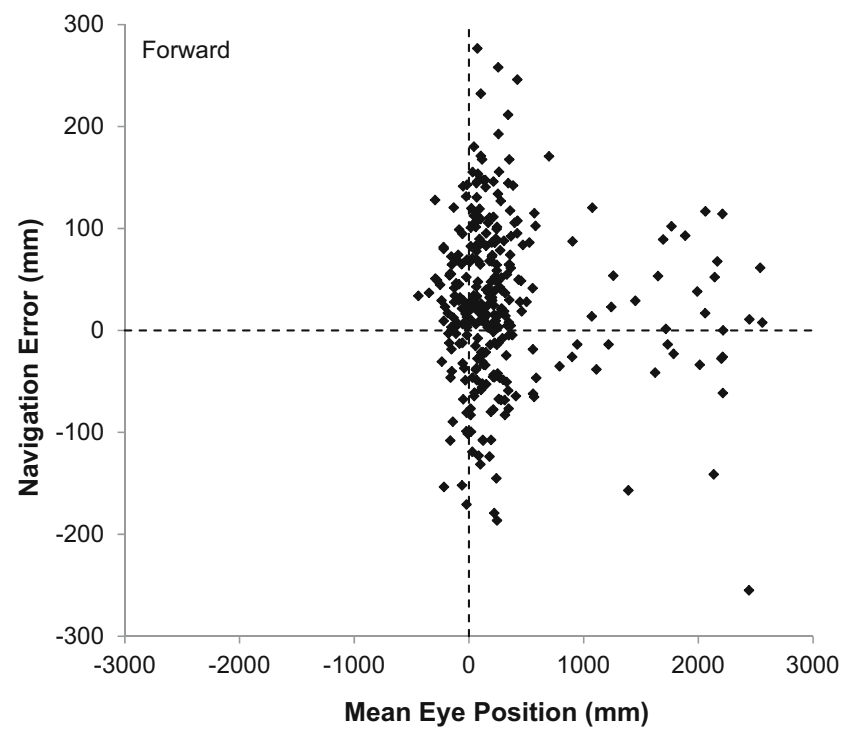

Fig. 11 Correlations between navigation errors and mean eye positions in the forward condition. Each data point represents one trial. Negative and positive values denote leftward and rightward deviations, respectively 
The rightward bias in eye fixations is consistent with a bisection-based theory of navigation asymmetry, whereby participants mentally "mark" the midpoint of a doorway as being toward the right and then head toward that point, resulting in rightward deviations and bumping. The rightward nature of participants' navigation errors and eye position is consistent with the existence of a rightward attentional bias for extrapersonal stimuli (Heber et al., 2010; Longo \& Lourenco, 2006; Nicholls et al., 2011; Nicholls, Loetscher, \& Rademacher, 2010).

It is noteworthy that individual participants demonstrated substantial differences in the degrees and, to a lesser extent, the directions of their typical gaze positions (see Figs. 6 and 7). In some cases, the deviation in gaze from the center of the aperture was quite substantial. Although these extreme rightward biases were unexpected, we have a number of reasons to believe that they were real. First, it should be noted that, whereas Figs. 8 and 9 suggest that some participants consistently looked to the far right of the doorway, each point in those figures was averaged over a large number of fixations. It is therefore likely that some fixations were made toward the aperture or the wheelchair, despite the fact that the majority of fixations fell far to the right. In addition, the eyetracker was calibrated before and after each session to ensure that the eyetracker and participant were aligned with the aperture. Given this alignment, the large deviations for some participants cannot be due to a misalignment of the eyetracking equipment. It is therefore likely that the variations in eye position reflect individual differences, which are known to affect attention and are reflected in tasks such as line bisection (Hach \& Schütz-Bosbach, 2012; Halligan, Manning, \& Marshall, 1990; McCourt \& Olafson, 1997; Rueckert, Deravanesian, Baboorian, Lacalamita, \& Repplinger, 2002). Since the presence of individual variation in bisection errors implies the existence of individual variation in attentional biases, these should also be reflected in eye movement biases, because these are tightly coupled with attention (Hoffman \& Subramaniam, 1995; Moore \& Fallah, 2001; Shepherd, Findlay, \& Hockey, 1986).

Surprisingly, we found little evidence of a relationship between participants' navigation errors and eye position. This finding may indicate that asymmetries in navigation and in eye movements do not reflect a common underlying attentional asymmetry. Inspection of Figs. 10 and 11, however, reveals very few trials on which the navigation error and eye position were both leftward. In future studies, selection of individuals with leftward attentional asymmetries may facilitate observation of a correlation between navigation errors and eye movements. Additionally, an individual's "navigation error" in this experiment comprised a single measurement taken at the end of each navigation trial, whereas the deviation score obtained for eye position was an aggregate reflecting the entire time course of each trial. It would be interesting in future studies to map the trajectory of a vehicle as it moves toward and through a doorway and to investigate whether eye position is related to the position of the vehicle at different time points within the trial.

Finally, the effect of cultural habits related to road rules needs to be considered. In a previous study by our group (Nicholls, Hadgraft, et al., 2010), we argued that road rules could not explain the rightward deviation. This argument was based on the premise that Australians, who drive on the left side of road, naturally keep to this side when navigating through an aperture - the opposite to what has been found for Australian participants (Nicholls, Hadgraft, et al., 2010; Nicholls et al., 2007; Nicholls et al., 2008). Although this argument seems reasonable, it remains to be tested explicitly by comparing the effects of different road rules on navigation asymmetries within the one study. A number of different studies, however, have used drivers with opposite driving habits. For drivers used to driving on the left side of the road, rightward biases have been reported by Nicholls and colleagues for ambulatory (Nicholls et al., 2007; Nicholls et al., 2008) and wheelchair (Nicholls, Hadgraft, et al., 2010) tasks in Australian drivers. In Japan, where drivers also keep to the left, some weak evidence of a rightward bias was observed for an ambulatory task - but this was heavily influenced by the foot used when walking through the doorway (Fujikake et al., 2011).

For drivers used to driving on the right side of the road, a rightward deviation has been reported by Jang et al. (2009) for Koreans controlling a car in a simulator. A rightward bias has also been reported for a virtual route-following task when it was presented in the upper visual hemispace for a group of North Americans. Conversely, when the task was presented in the lower hemispace, a leftward bias was observed (Thomas et al., 2009). Finally, more leftward collisions have been reported by Hatin et al. (2012) for an ambulatory task in a North American population.

As can be seen, the data related to driving direction are not entirely clear. For populations used to driving on the left side of the road, the rightward deviation appears to be relatively consistent. Experiments conducted with populations driving on the right side of the road are perhaps less clear; although two of the studies showed some evidence of a rightward bias, another study showed a leftward bias.

Another means of examining the effect of driving direction would be to compare studies that have examined asymmetries in attention and eye direction during a navigation task. Although the present study has demonstrated that Australian participants direct their gaze to the right when navigating a wheelchair though a doorway, the opposite has been reported by Cinelli et al. (2009) for a North American population for an ambulatory task. Cinelli et al. attributed the predominance of leftward fixations during their task to the fact that their North American participants drove on the right side and were 
therefore used to attending to the left as they passed an upcoming car/obstacle. A leftward bias of attention has also been observed by Benedetto, Pedrotti, Bremond, and Baccino (2013) for a simulated driving task in French drivers, who also drive on the right. The attention/ eye fixation data therefore seem consistent with a cultural effect of driving direction.

In the present study, we attempted to examine the effect of driving habits by comparing deviations in the trajectory of a wheelchair and in eye movements as participants maneuvered the wheelchair through a doorway. It was argued that participants might "imagine" themselves in the wheelchair as they maneuvered it. If this were the case - and the bias was the result of road rules - one might have expected the deviation to reverse when the wheelchair was reversed. This reversal was not observed. It must be acknowledged, however, that this proposal was based on a number of assumptions, which have not been directly tested. The effect of road rules therefore remains as an interesting and potentially important factor to be tested in studies of navigation asymmetries.

Author note We extend thanks to Lauren Nicholls for constructing Fig. 1 and to Max Rademacher for helping to modify the wheelchair.

\section{References}

Aimola, L., Schindler, I., Simone, A. M., \& Venneri, A. (2012). Near and far space neglect: Task sensitivity and anatomical substrates. Neuropsychologia, 50, 1115-1123. doi:10.1016/j. neuropsychologia.2012.01.022

Azouvi, P., Marchal, F., Samuel, C., Morin, L., Renard, C., LouisDreyfus, A., ... Bergego, C. (1996). Functional consequences and awareness of unilateral neglect: Study of an evaluation scale. Neuropsychological Rehabilitation, 6, 133-150. doi:10.1080/ 713755501

Baek, M. J., Lee, B. H., Kwon, J. C., Park, J. M., Kang, S. J., Chin, J., .. $\mathrm{Na}$, D. L. (2002). Influence of final search direction on tactile line bisection in normal subjects. Neurology, 58, 1833-1838. doi:10. 1212/WNL.58.12.1833

Bartolomeo, P., \& Chokron, S. (1999). Left unilateral neglect or right hyperattention? Neurology, 53, 2023-2027. doi:10.1212/WNL.53. 9.2023

Beauchamp, M. S., Petit, L., Ellmore, T. M., Ingeholm, J., \& Haxby, J. V. (2001). A parametric fMRI study of overt and covert shifts of visuospatial attention. NeuroImage, 14, 310-321. doi:10.1006/ nimg.2001.0788

Benedetto, S., Pedrotti, M., Bremond, R., \& Baccino, T. (2013). Leftward attentional bias in a simulated driving task. Transport Research Part F: Traffic Psychology and Behavior, 20, 147-153. doi:10.1016/j.trf. 2013.07.006

Berti, A., Smania, N., Rabuffetti, M., Ferrarin, M., Spinazzola, L., D'Amico, A., ... Allport, A. (2002). Coding of near and far space during walking in neglect patients. Neuropsychology, 16, 390-399. doi:10.1037/0894-4105.16.3.390

Bisiach, E., \& Vallar, G. (1988). Hemineglect in humans. In F. Boller, J. Grafman, G. Rizzolatti, \& H. Goodglass (Eds.), Handbook of neuropsychology (Vol. 1, pp. 195-222). New York, NY: Elsevier Science.
Bowers, D., \& Heilman, K. M. (1980). Pseudoneglect: Effects of hemispace on a tactile line bisection task. Neuropsychologia, 18, 491-498. doi:10.1016/0028-3932(80)90151-7

Boyadjian, A., Main, L., \& Danion, F. (1999). Veering in human locomotion: The role of effectors. Neuroscience Letters, 265, 21-24. doi: 10.1016/S0304-3940(99)00198-6

Bradshaw, J. L., Bradshaw, J. A., Nathan, G., Nettleton, N. C., \& Wilson, L. E. (1986). Leftwards error in bisecting the gap between two points: Stimulus quality and hand effects. Neuropsychologia, 24, 849-855. doi:10.1016/0028-3932(86)90084-9

Brain, W. R. (1941). Visual disorientation with special reference to lesions of the right cerebral hemisphere. Brain, 64, 244-272. doi: 10.1093/brain/64.4.244

Brainard, D. H. (1997). The Psychophysics Toolbox. Spatial Vision, 10, 433-436. doi:10.1163/156856897X00357

Brozzoli, C., Dematte, M. L., Pavani, F., Frassinetti, F., \& Farnè, A. (2006). Neglect and extinction: Within and between sensory modalities. Restorative Neurology and Neuroscience, 24, 217-232.

Bultitude, J. H., \& Aimola Davies, A. M. (2006). Putting attention on the line: Investigating the activation-orientation hypothesis of pseudoneglect. Neuropsychologia, 44, 346-358. doi:10.1016/j. neuropsychologia.2006.03.001

Chokron, S., Bartolomeo, P., Perenin, M., Helft, G., \& Imbert, M. (1998). Scanning direction and line bisection: A study of normal subjects and unilateral neglect patients with opposite reading habits. Cognitive Brain Research, 7, 173-178. doi:10.1016/S09266410(98)00022-6

Chokron, S., \& Imbert, M. (1993). Influence of reading habits on line bisection. Cognitive Brain Research, 1, 219-222. doi:10.1016/ 0926-6410(93)90005-P

Cinelli, M. E., Patla, A. E., \& Allard, F. (2009). Behavior and gaze analyses during a goal-directed locomotor task. Quarterly Journal of Experimental Psychology, 62, 483-499. doi:10.1080/ 17470210802168583

Cohen, H. S. (2000). Vestibular disorders and impaired path integration along a linear trajectory. Journal of Vestibular Research, 10, 7-15.

de Haan, B., Karnath, H. O., \& Driver, J. (2012). Mechanisms and anatomy of unilateral extinction after brain injury. Neuropsychologia, 50, 1045-1053. doi:10.1016/j.neuropsychologia.2012.02.015

Deubel, H., \& Schneider, W. X. (1996). Saccade target selection and object recognition: Evidence for a common attentional mechanism. Vision Research, 36, 1827-1837. doi:10.1016/0042-6989(95) 00294-4

Drain, M., \& Reuter-Lorenz, P. A. (1996). Vertical orienting control: Evidence for attentional bias and "neglect" in the intact brain. Journal of Experimental Psychology: General, 125, 139-158. doi: 10.1037/0096-3445.125.2.139

Fujikake, H., Higuchi, T., Imanaka, K., \& Maloney, L. (2011). Directional bias in the body while walking through a doorway: Its association with attentional and motor factors. Experimental Brain Research, 210, 195-206. doi:10.1007/s00221-011-2621-3

Gamberini, L., Seraglia, B., \& Priftis, K. (2008). Processing of peripersonal and extrapersonal space using tools: Evidence from visual line bisection in real and virtual environments. Neuropsychologia, 46, 1298-1304. doi:10.1016/j.neuropsychologia. 2007.12.016

Hach, S., \& Schütz-Bosbach, S. (2012). Touching base: The effect of participant and stimulus modulation factors on a haptic line bisection task. Laterality, 17, 180-201. doi:10.1080/1357650X.2010.551128

Halligan, P. W., Manning, L., \& Marshall, J. C. (1990). Individual variation in line bisection: A study of four patients with right hemisphere damage and normal controls. Neuropsychologia, 28, 1043-1051. doi:10.1016/0028-3932(90)90139-F

Hatin, B., Tottenham, L. S., \& Oriet, C. (2012). The relationship between collisions and pseudoneglect: Is it right? Cortex, 48, 997-1008. doi: 10.1016/j.cortex.2011.05.015 
Heber, I. A., Siebertz, S., Wolter, M., Kuhlen, T., \& Fimm, B. (2010). Horizontal and vertical pseudoneglect in peri- and extrapersonal space. Brain and Cognition, 73, 160-166. doi:10.1016/j.bandc. 2010.04.006

Heilman, K. M., Jeong, Y., \& Finney, G. R. (2004). Spatial cognition. Neurology, 63, 1994-1996. doi:10.1212/01.WNL.0000146888. 37987.48

Heilman, K. M., \& Valenstein, E. (1979). Mechanisms underlying hemispatial neglect. Annals of Neurology, 5, 166-170. doi:10. 1002/ana.410050210

Heilman, K. M., Watson, R. T., \& Valenstein, E. (2003). Neglect and related disorders. In K. Heilman \& E. Valenstein (Eds.), Clinical neuropsychology (4th ed., pp. 296-346). New York, NY: Oxford University Press.

Hoffman, J. E., \& Subramaniam, B. (1995). The role of visual attention in saccadic eye movements. Perception and Psychophysics, 57, 787795. doi:10.3758/BF03206794

Hornak, J. (1992). Ocular exploration in the dark by patients with visual neglect. Neuropsychologia, 30, 547-552. doi:10.1016/00283932(92) $90057-\mathrm{S}$

Jackson, D., Thornton, H., \& Turner-Stokes, L. (2000). Can young severely disabled stroke patients regain the ability to walk independently more than three months post stroke? Clinical Rehabilitation, 14, 538-547. doi:10.1191/0269215500cr358oa

Jang, S.-L., Ku, B.-D., Na, D.-L., \& Lee, J.-H. (2009). Effects of pseudoneglect in visual attention: Evidence for car laterality using a driving simulator. CyberPsychology and Behavior, 12, 81-112. doi:10.1089/cpb.2008.9999.abs

Jewell, G., \& McCourt, M. E. (2000). Pseudoneglect: A review and metaanalysis of performance factors in line bisection tasks. Neuropsychologia, 38, 93-110. doi:10.1016/S0028-3932(99)00045-7

Karnath, H. O., \& Fetter, M. (1995). Ocular space exploration in the dark and its relation to subjective and objective body orientation in neglect patients with parietal lesions. Neuropsychologia, 33, 371377. doi:10.1016/0028-3932(94)00115-6

Kim, D. Y., Ku, J., Chang, W. H., Park, T. H., Lim, J. Y., Han, K., ... Kim, S. I. (2010). Assessment of post-stroke extrapersonal neglect using a three-dimensional immersive virtual street crossing program. Acta Neurologica Scandinavica, 121, 171-177. doi:10.1111/j.16000404.2009.01194.x

Kinsbourne, M. (1970). The cerebral basis of lateral asymmetries in attention. Acta Psychologica, 33, 193-201. doi:10.1016/00016918(70)90132-0

Kinsbourne, M. (1977). Hemi-neglect and hemisphere rivalry. Advances in Neurology, 18, 41-49.

Kinsbourne, M. (1987). Mechanisms of unilateral neglect. In M. Jeannerod (Ed.), Neurophysiological and neuropsychological aspects of spatial neglect (pp. 69-113). Amsterdam, The Netherlands: Elsevier Science.

Longo, M. R., \& Lourenco, S. F. (2006). On the nature of near space: Effects of tool use and the transition to far space. Neuropsychologia, 44, 977-981. doi:10.1016/j.neuropsychologia.2005.09.003

Luh, K. (1995). Line bisection and perceptual asymmetries in normal individuals: What you see is not what you get. Neuropsychology, 9, 435-448. doi:10.1037/0894-4105.9.4.435

Mattingley, J. B., Berberovic, N., Corben, L., Slavin, M. J., Nicholls, M. E. R., \& Bradshaw, J. L. (2004). The greyscales task: A perceptual measure of attentional bias following unilateral hemispheric damage. Neuropsychologia, 42, 387-394. doi:10.1016/j.neuropsychologia. 2003.07.007

Mayo, N. E., Wood-Dauphinee, S., Ahmed, S., Gordon, C., Higgins, J., McEwen, S., \& Salbach, N. (1999). Disablement following stroke. Disability and Rehabilitation, 21, 258-268.

McCourt, M. E. (2001). Performance consistency of normal observers in forced-choice tachistoscopic visual line bisection. Neuropsychologia, 39, 1065-1076. doi:10.1016/S0028-3932(01)00044-6
McCourt, M. E., \& Jewell, G. (1999). Visuospatial attention in line bisection: Stimulus modulation of pseudoneglect. Neuropsychologia, 37, 843-855. doi:10.1016/S0028-3932(98) 00140-7

McCourt, M. E., \& Olafson, C. (1997). Cognitive and perceptual influences on visual line bisection: Psychophysical and chronometric analyses of pseudoneglect. Neuropsychologia, 17, 1194-1211. doi: 10.1016/S0028-3932(96)00143-1

Mohr, C., Landis, T., Bracha, H., Fathi, M., \& Brugger, P. (2003). Human locomotion: Levodopa keeps you straight. Neuroscience Letters, 339, 115-118. doi:10.1016/S0304-3940(02)01480-5

Mohr, C., Landis, T., Bracha, H. S., Fathi, M., \& Brugger, P. (2005). Levodopa reverses gait asymmetries related to anhedonia and magical ideation. European Archives of Psychiatry and Clinical Neuroscience, 255, 33-39. doi:10.1007/s00406-004-0531-0

Molenberghs, P., Sale, M. V., \& Mattingley, J. B. (2012). Is there a critical lesion site for unilateral spatial neglect? A meta-analysis using activation likelihood estimation. Frontiers in Human Neuroscience, 6, 78. doi:10.3389/fnhum.2012.00078

Moore, T., \& Fallah, M. (2001). Control of eye movements and spatial attention. Proceedings of the National Academy of Sciences, 98, $1273-1276$.

Nicholls, M. E. R., Bradshaw, J. L., \& Mattingley, J. B. (1999). Freeviewing perceptual asymmetries for the judgement of brightness, numerosity and size. Neuropsychologia, 37, 307-314. doi:10.1016/ S0028-3932(98)00074-8

Nicholls, M. E. R., Forte, J. D., Loetscher, T., Orr, C. A., Yates, M. J., \& Bradshaw, J. L. (2011). Near, yet so far: The effect of pictorial cues on spatial attention. Brain and Cognition, 76, 349-352. doi:10. 1016/j.bandc.2011.04.006

Nicholls, M. E. R., Hadgraft, N., Chapman, H., Loftus, A. M., Robertson, J. S., \& Bradshaw, J. L. (2010a). A hit and miss investigation of asymmetries in wheelchair navigation. Attention, Perception, \& Psychophysics, 72, 1576-1590. doi:10.3758/APP.72.6.1576

Nicholls, M. E. R., Loetscher, T., \& Rademacher, M. (2010b). Miss to the right: The effect of attentional asymmetries on goal-kicking. PLoS ONE, 5, e12363. doi:10.1371/journal.pone.0012363

Nicholls, M. E. R., Loftus, A. M., Mayer, K., \& Mattingley, J. B. (2007). Things that go bump in the right: The effect of unimanual activity on rightward collisions. Neuropsychologia, 45, 1122-1126. doi:10. 1016/j.neuropsychologia.2006.07.015

Nicholls, M. E. R., Loftus, A. M., Orr, C. A., \& Barre, N. (2008) Rightward collisions and their association with pseudoneglect. Brain and Cognition, 68, 166-170. doi:10.1016/j.bandc.2008.04.003

Nicholls, M. E. R., Mattingley, J. B., \& Bradshaw, J. L. (2005). The effect of strategy on pseudoneglect for luminance judgements. Cognitive Brain Research, 25, 71-77.

Nicholls, M. E. R., \& Roberts, G. R. (2002). Can free-viewing perceptual asymmetries be explained by scanning, pre-motor or attentional biases? Cortex, 38, 113-136. doi:10.1016/S0010-9452(08)70645-2

Nicholls, M. E. R., Smith, A., Mattingley, J. B., \& Bradshaw, J. L. (2006). The effect of body and environment-centred coordinates on freeviewing perceptual asymmetries for vertical and horizontal stimuli. Cortex, 42, 336-346. doi:10.1016/S0010-9452(08)70360-5

Oldfield, R. C. (1971). The assessment and analysis of handedness: The Edinburgh inventory. Neuropsychologia, 9, 97-113. doi:10.1016/ 0028-3932(71)90067-4

Punt, T. D., Kitadono, K., Hulleman, J., Humphreys, G. W., \& Riddoch, M. J. (2008). From both sides now: Crossover effects influence navigation in patients with unilateral neglect. Journal of Neurology, Neurosurgery, and Psychiatry, 79, 464-466. doi:10. 1136/innp.2007.129205

Punt, T. D., Kitadono, K., Hulleman, J., Humphreys, G. W., \& Riddoch, M. J. (2011). Modulating wheelchair navigation in patients with spatial neglect. Neuropsychological Rehabilitation, 21, 367-382. doi:10.1080/09602011.2011.559132 
Qiang, W., Sonoda, S., Suzuki, M., Okamoto, S., \& Saitoh, E. (2005). Reliability and validity of a wheelchair collision test for screening behavioral assessment of unilateral neglect after stroke. American Journal of Physical Medicine and Rehabilitation, 84, 161-166. doi: 10.1097/01.PHM.0000154902.79990.12

Roberts, J. C., Cohen, H. S., \& Sangi-Haghpeykar, H. (2011). Vestibular disorders and dual task performance: Impairment when walking a straight path. Journal of Vestibular Research, 21, 167-174. doi:10. 3233/VES-2011-0415

Robertson, I. H., \& Halligan, P. W. (1999). Spatial neglect: A clinical handbook for diagnosis and treatment. Hove, UK: Psychology Press.

Robertson, I. H., Tegnér, R., Goodrich, S. J., \& Wilson, C. (1994). Walking trajectory and unimanual movements in unilateral left neglect: A vestibular hypothesis. Neuropsychologia, 32, 14951502. doi:10.1016/0028-3932(94)90121-X

Rueckert, L., Deravanesian, A., Baboorian, D., Lacalamita, A., \& Repplinger, M. (2002). Pseudoneglect and the cross-over effect. Neuropsychologia, 40, 162-173. doi:10.1016/S00283932(01)00082-3

Shepherd, M., Findlay, J. M., \& Hockey, R. J. (1986). The relationship between eye movements and spatial attention. Quarterly Journal of Experimental Psychology, 38A(3), 475-491. doi:10.1080/ 14640748608401609

Thomas, N. A., Stuckel, D., Gutwin, C., \& Elias, L. J. (2009). Directional collisions during a route-following task. Journal of the International Neuropsychological Society, 15, 225-230. doi:10. 1017/S1355617709090328

Toth, C., \& Kirk, A. (2002). Representational bias does not affect bisection of lines with a pictorially or semantically defined top by patients with left hemispatial neglect. Brain and Cognition, 50, 167-177.
Turnbull, O. H., \& McGeorge, P. (1998). Lateral bumping: A normalsubject analog to the behaviour of patients with hemispatial neglect? Brain and Cognition, 37, 31-33.

Turton, A. J., Dewar, S. J., Lievesley, A., O'Leary, K., Gabb, J., \& Gilchrist, I. D. (2009). Walking and wheelchair navigation in patients with left visual neglect. Neuropsychological Rehabilitation, 19, 274-290. doi:10.1080/09602010802106478

Vallar, G., Guariglia, C., Nico, D., \& Bisiach, E. (1995). Spatial hemineglect in back space. Brain, 118, 467-472. doi:10.1093/brain/118.2.467

Verfaellie, M., \& Heilman, K. M. (2005). Neglect syndromes. In P. J. Snyder, P. D. Nussbaum, \& D. L. Robins (Eds.), Clinical neuropsychology: A pocket handbook for assessment (pp. 489-507). Washington, DC: American Psychological Association.

Webster, J. S., Cottam, G., Gouvier, W., Blanton, P., Beissel, G., \& Wofford, J. (1989). Wheelchair obstacle course performance in right cerebral vascular accident victims. Journal of Clinical and Experimental Neuropsychology, 11, 295-311.

Webster, J. S., Rapport, L. J., Godlewski, M. C., \& Abadee, P. S. (1994). Effect of attentional bias to right space on wheelchair mobility. Journal of Clinical and Experimental Psychology, 16, 129-137. doi:10.1080/01688639408402623

Webster, J. S., Roads, L. A., Morrill, B., Rapport, L. J., Abadee, P. S., Sowa, M. V., ... Godlewski, M. C. (1995). Rightward orienting bias, wheelchair maneuvering and fall risk. Archives of Physical Medicine and Rehabilitation, 76, 924-928. doi:10.1016/S0003-9993(95)80068-9

Weintraub, S., \& Mesulam, M. M. (1987). Right cerebral dominance in spatial attention. Archives of Neurology, 44, 621-625.

Weintraub, S., \& Mesulam, M. M. (1988). Visual hemispatial inattention: Stimulus parameters and exploratory strategies. Journal of Neurology, Neurosurgery, and Psychiatry, 51, 1481-1488. doi:10. 1136/jnnp.51.12.1481 